\title{
The Crystal and Molecular Structure of the Nematogenic Compound N-p-Methoxy- benzylidene-p-phenylazoaniline (MBPAA)
}

\author{
BY G. V. VANI \\ Raman Research Institute, Bangalore 560006, India \\ AND KALYANi ViJAYAN \\ Materials Science Division, National Aeronautical Laboratory, Bangalore 560017, India
}

(Received 4 October 1976; accepted 13 January 1977)

\begin{abstract}
The crystalline form of $N$-p-methoxybenzylidine-p-phenylazoaniline (MBPAA) has a monoclinic unit cell, space group $P 2_{1} / c$, with eight molecules per cell. The cell parameters are $a=9.77$ (1), $b=23.65(2), c=$ 15.64 (1) $\AA$ and $\beta=112.4(3)^{\circ}$. The crystal structure was solved by a combination of direct methods, packing considerations and trial calculations, and has been refined by block-diagonal least-squares calculations to $R=0.126$ for 1071 photographically measured reflexions. One of the crystallographically independent molecules in the asymmetric part has been found to have partial positional disorder due to the existence of two rotational conformers of that part of the molecule, occurring with unequal weights. In the crystal structure neighbouring molecules are oriented antiparallel to each other and the molecular arrangement corresponds to a herringbone-like pattern.
\end{abstract}

\section{Introduction}

A detailed knowledge of the structural features of mesogenic substances in their crystalline phase is of considerable value in understanding their structure and properties in the mesophase. We have, therefore, undertaken a programme of single-crystal X-ray studies on mesogenic substances. As part of this programme, the crystal and molecular structure of a nematogenic

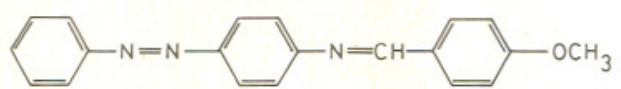

(I) compound, $N$-p-methoxybenzylidene- $p$-phenylazoaniline (MBPAA), (I) has been determined. A preliminary account of this work was reported in the Proceedings of the International Liquid Crystals Conference, held at Bangalore (Vijayan \& Vani, 1975).

Table 1. Crystal data

$$
\begin{array}{ll}
\mathrm{C}_{20} \mathrm{H}_{17} \mathrm{ON}_{3}, M_{r}=315 \cdot 38 & \text { Space group } P 2_{1} / c \\
a=9 \cdot 77(1) \AA & Z=8 \\
b=23.65(2) & D_{c}=1.25 \mathrm{~g} \mathrm{~cm}^{-3} \\
c=15.64(1) & D_{m} \text { (flotation) }=1.26 \\
\beta=112.4(3)^{\circ} &
\end{array}
$$





\section{Experimental}

Orange-coloured needles of MBPAA were obtained by slow evaporation from a solution in toluene. The space group and unit-cell dimensions as determined from oscillation and Weissenberg photographs are presented in Table 1 along with other relevant crystal data. Threedimensional intensity data from the reciprocal levels $h k 0$ and $H k l, H=0$ to 5 , were recorded on multiple films by the equi-inclination Weissenberg method using $\mathrm{Ni}$-filtered $\mathrm{Cu}$ radiation. Even though the crystals were optically clear, the diffraction pattern was of poor quality. In particular, the data faded off rapidly with increasing Bragg angle. Out of a total of 7717 possible independent reflexions in the $\mathrm{Cu} K \alpha$ sphere only 1071 were in the observable range with a maximum Bragg angle of $57.8^{\circ}$. The intensities were corrected for Lorentz-polarization factors, absorption and spotshape effects.

\section{Structure analysis}

The chemical formula indicated the molecules to be nearly planar and elongated and, hence, the distribution of atoms in the unit cell was expected to be highly nonrandom. This feature, together with the absence of high-angle data led us to try trial-and-error and Patterson techniques of structure analysis before attempting to apply the conventional direct methods to solve this equal-atom structure.

An examination of the diffraction data revealed that triple-product relationships existed among the nine strongest low-angle reflexions in the pattern. A 'mini' symbolic addition procedure involving these nine reflexions was then carried out, using two symbols. The four possible Fourier maps with nine reflexions were very similar in character and indicated that the two crystallographically independent molecules in the structure lay nearly in the $b c$ plane with a separation of $a / 2$ between them. Even though the molecular orientations indicated in these maps were different, they were independently compatible with the vector distribution in the threedimensional Patterson function.

Sign determination using MULTAN (Germain, Main \& Woolfson, 1971), modified for the IBM 360/44 computer, was next attempted. The best solution gave a distribution of peaks which appeared to give geometrically feasible positions to the molecules, but the atomic positions could not, however, be fixed.

$E$ values were recalculated using molecular scattering factors for spherically averaged phenyl rings. From the $E$ map corresponding to the best solution of the new MULTAN, the atomic positions of one of the crystallographically independent molecules could be fixed and this partial solution agreed well with one of the ninereflexion Fourier maps mentioned earlier. The atomic positions in the other molecule were not clear and could not be derived from the subsequent difference Fourier map either. However, by superposing the features of the nine-reflexion Fourier map on the $E$ map, two trial models were fixed for the second molecule. One of the models proved to be correct with an initial $R$ value of 0.519 , which reduced to 0.146 in successive blockdiagonal least-squares refinement. The weighting scheme used was of the form $w=1 /\left(a+b F_{o}+c F_{o}^{2}\right)$ with $a=0.70, b=-0.05$ and $c=-0.009$. All atoms were treated with isotropic thermal vibration parameters. On account of the low value of the reflexion-to-parameter ratio, arising from the extreme paucity of data, refinement of anisotropic thermal parameters was not attempted. The scattering factors used were those of Cromer \& Waber (1965). The SFLS program was written originally by Shiono and modified for the IBM 360/44 computer by Dr B. S. Reddy.

\section{Disorder}

In the course of the least-squares refinement, the $B$ values of $\mathrm{C}(25)$ through $\mathrm{C}(48)$, referred to as molecule $B$ hereinafter, were found to be generally higher than those of $\mathrm{C}(1)$ through $\mathrm{C}(24)$, referred to as molecule $A$. (The numbering of atoms is shown in Fig. 1.) In particular, the $B$ values of the azo $\mathrm{N}$ atoms $\mathrm{N}(31)$ and $\mathrm{N}(32)$ and the atoms of the phenyl rings on either side of the azo bond were conspicuously high. The $B$ values of $\mathrm{N}(31)$ and $\mathrm{N}(32)$ were the highest, being about $2-2.5$ times the average $B$ values for the rest of the structure. This feature led us to suspect the possibility of disorder in that region of molecule $B$. A difference Fourier map computed after removal of the contributions of the $\mathrm{N}$ atoms and the neighbouring aromatic $\mathrm{C}$ atoms clearly showed two extra positions for $\mathrm{N}(31)$ and $\mathrm{N}(32)$. For the ring atoms, however, even though two discrete sets of peaks were not observed, the diffuse electron density distribution allowed the possibility of a second set of atomic positions. The two models could be described as rotational conformers about the line joining the ring atoms $\mathrm{C}(28)$ and $\mathrm{C}(33)$. The relative heights of the two sets of peaks for the $\mathrm{N}$ atoms suggested that the rotational conformers occurred with unequal weights. Hence, the positional occupancy factors of the 14 atoms with double positions were subsequently refined by the least-squares procedure and were found to be 0.7 for the original set of atomic positions and 0.3 for the new set fixed from the difference map. Further refinement of the structure with individual isotropic thermal parameters was carried out including the two sets of atomic positions with the appropriate occupancy factors. It may be mentioned now that the molecule with positional disorder was the one which could not be identified from the $E$ map. When the least-squares refinement converged, the $R$ value was 0.126 for 1071 observed reflexions.

Similar positional disorder about the $-\mathrm{N}=\mathrm{N}-$ bond 
connecting two phenyl rings has been observed in the crystal structures of $p$-azotoluene (Brown, 1966b), $p$-azobenzene (Brown, 1966a) and the high-temperature form of smectogenic ethyl $p$-azoxybenzoate (Krigbaum \& Taga, 1974). We are carrying out a detailed study of the disorder about the $-\mathrm{N}=\mathrm{N}-$ bond in these structures and the results will be published elsewhere. Recently, Hoekstra, Meertens \& Vos (1975) have reported a similar feature about a $-\mathrm{C}=\mathrm{C}-$ bond connecting two phenyl rings in the crystal structure of stilbene.

\section{Results and description}

The final positional and thermal parameters are given in Table 2 and the interatomic distances and valency angles in Fig. 1.* As the least-squares refinement did not include the correlation between an atom and its disordered counterpart, the atomic parameters as well as the molecular dimensions in the disordered region have low reliability. The dimensions in molecule $A$ and in the ordered region of molecule $B$ are normal within the limits of experimental error.

The least-squares planes through various groups of atoms in the structure have been calculated with respect to an orthogonal set of axes. The aromatic rings and

* A list of structure factors has been deposited with the British Library Lending division as Supplementary Publication No. SUP 32445 (6 pp.). Copies may be obtained through The Executive Secretary. International Union of Crystallography, 13 White Friars, Chester CH1 1NZ, England.
Ring 1

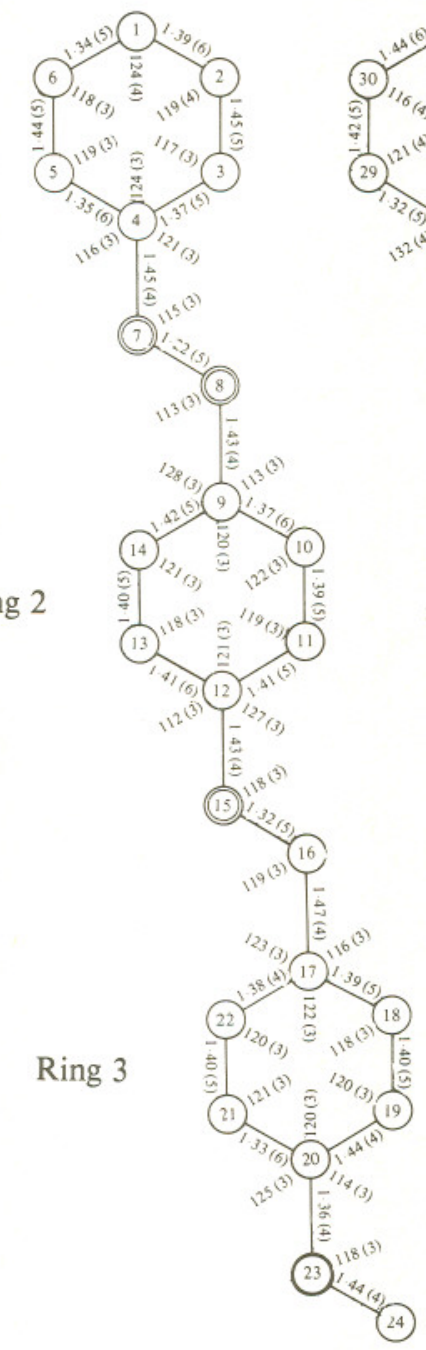

Molecule $A$

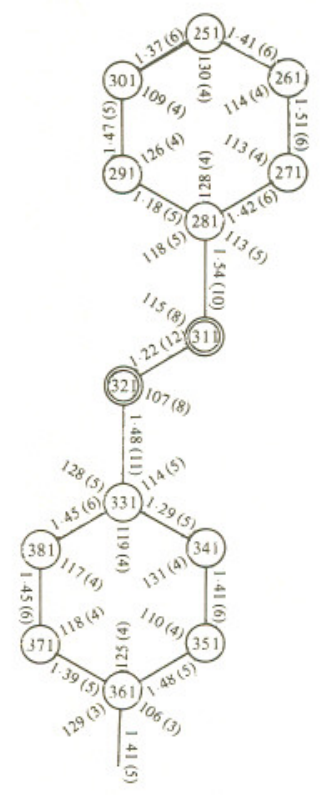

\section{Carbon}

(1) Nitrogen

\section{Molecule $B$}

Oxygen

Fig. 1. Bond lengths $(\AA)$ and bond angles $\left(^{\circ}\right)$ with e.s.d.'s in parentheses. The dimensions of the disordered region are less reliable (see text). The numbering of the rings is also shown. 
the azo groups in the structure are planar. In mclecule $A$, the angle between the plane normals of rings 1 and 2 (ring numbering shown in Fig. 1) is $3^{\circ}$ and ring 3 is inclined to rings 1 and 2 by 21 and $19^{\circ}$ respectively. The averages of the corresponding values for the disordered molecule $B$ are $8,-33$ and $-26^{\circ}$ respectively.

The arrangement of the molecules in a plane perpendicular to the $a$ axis is shown in Fig. 2. The crystallographically independent molecules $A$ and $B$ lie almost exactly above each other with a separation of about $5 \AA$ along a. They are oriented antiparallel to each other. The slightly overlapping phenyl rings in molecules $A$ and $B$ are not oriented parallel but are inclined with respect to one another, the inclination being $58^{\circ}$ at one end, changing to $99^{\circ}$ near the molecular centre and $108^{\circ}$ at the other end. To describe the packing of molecules in the crystal, it is convenient to consider as one unit the crystallographically independent molecules and their symmetry equivalents generated by a centre of inversion at the origin. This unit is indicated in Fig. 2. The two pairs of molecules in this quadruple unit are oriented antiparallel to each other. The quadruple unit is inclined to the $c$ axis at about $55^{\circ}$. Hence, the neighbouring units related by the $c$-cell translation are so displaced that the end of one unit falls near the centre of the neighbouring unit, forming an infinite column of quadruple molecular units arranged in an imbricated fashion along the $c$ axis. Along $b$, adjacent imbricated columns are related by the 2, screw axis and the $c$ glide. The molecular units in adjacent columns are inclined to each other at about $70^{\circ}$ and form a herring-

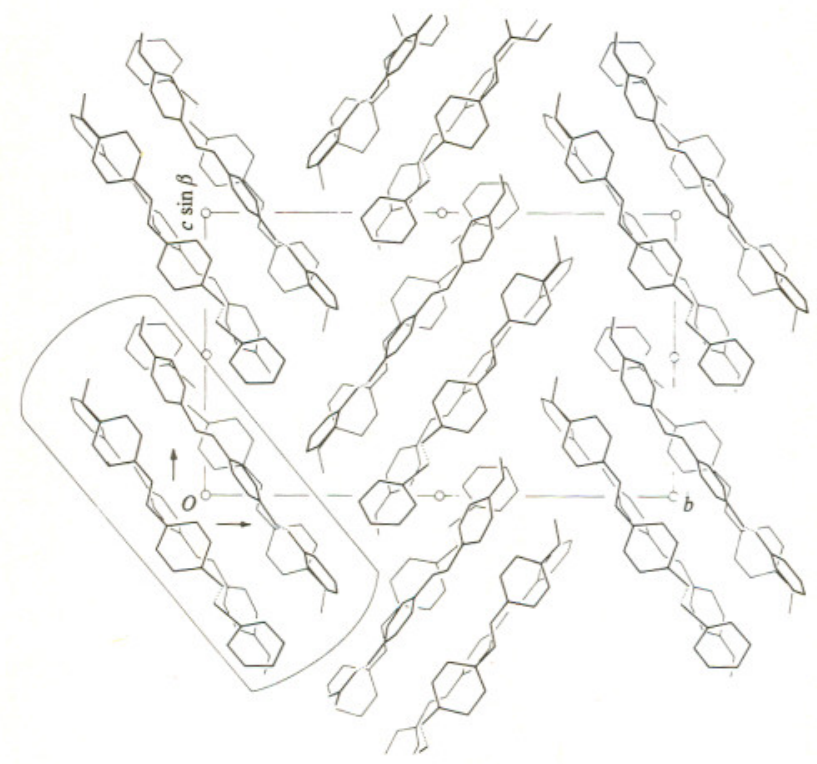

Fig. 2. View of the crystal structure perpendicular to the $a$ axis. The dotted lines represent the $-\mathrm{N}=\mathrm{N}-$ bond. The basic, quadruple molecular unit used for describing the packing has also been indicated. In the disordered region only the positions with 0.7 occupancy have been marked.
Table 2. Final fractional positional $\left(\times 10^{3}\right)$ coordinates and thermal parameters with e.s.d.'s in parentheses

$\mathrm{C}(1)$
$\mathrm{C}(2)$

$\mathrm{C}(3)$

$\mathrm{C}(4)$

$\mathrm{C}(5)$

$\mathrm{C}(6)$

$\mathrm{N}(7)$

$\mathrm{N}(8)$

$\mathrm{C}(9)$

$\mathrm{C}(10)$

$\mathrm{C}(11)$

$\mathrm{C}(12)$

C(13)

C(14)

$\mathrm{N}(15)$

C(16)

C(17)

C(18)

C(19)

C(20)

C(21)

$\mathrm{C}(22)$

$\mathrm{O}(23)$

C(24)

C (25)

C (26)

$\mathrm{C}(27)$

C(28)

C(29)

C(30)

$\mathrm{N}(31)$

$\mathrm{N}(32)$

C(33)

C(34)

C(35)

C(36)

C(37)

C(38)

$\mathrm{N}(39)$

$\mathrm{C}(40)$

C(41)

C (42)

C(43)

C(44)

C(45)

C(46)

$\mathrm{O}(47)$

C(48)

C (251)

C(261)

C(271)

C(281)

C (291)

C(301)

$\mathrm{N}(311)$

$\mathrm{N}(321)$

C(331)

C(341)

C(351)

$\mathrm{C}(361)$

C(371)

C(381)

\begin{tabular}{|c|c|c|c|}
\hline$x$ & $y$ & $z$ & $B\left(\AA^{2}\right)$ \\
\hline 916 (4) & $282(1)$ & $-345(2)$ & $5 \cdot 1(0.9)$ \\
\hline $1028(5)$ & $258(2)$ & $-323(3)$ & $6.4(1.0)$ \\
\hline 987 (4) & 221 (1) & $-245(2)$ & $4.2(0.8)$ \\
\hline 838 (4) & 212 (1) & $-196(2)$ & $3.4(0.7)$ \\
\hline 731 (4) & 236 (1) & -219 (2) & $5.3(0.8)$ \\
\hline 772 (4) & 273 (1) & $-298(2)$ & $5.4(0.9)$ \\
\hline 788 (3) & $174(1)$ & $-117(2)$ & $4.7(0.6)$ \\
\hline 885 (3) & 152 (1) & $-98(2)$ & $4.9(0.7)$ \\
\hline 829 (4) & $116(1)$ & $-19(2)$ & $4.2(0.8)$ \\
\hline 939 (4) & 93 (1) & $3(2)$ & $4.4(0.8)$ \\
\hline 907 (4) & 60 (1) & $83(2)$ & $4.7(0.8)$ \\
\hline $758(4)$ & 48 (1) & $138(2)$ & $5.7(0.9)$ \\
\hline 642 (4) & 72 (1) & $117(2)$ & $3.8(0.7)$ \\
\hline 679 (4) & 107 (1) & $40(2)$ & $5.1(0.9)$ \\
\hline 704 (3) & 14 (1) & $220(2)$ & $5.0(0.7)$ \\
\hline 798 (4) & $-20(1)$ & $234(2)$ & $4.9(0.8)$ \\
\hline 749 (4) & $-52(1)$ & $321(2)$ & $4.2(0.7)$ \\
\hline 854 (4) & $-87(1)$ & $333(2)$ & $4.0(0.8)$ \\
\hline 816 (4) & $-116(1)$ & $418(2)$ & $5.0(0.9)$ \\
\hline $670(4)$ & $-110(1)$ & $487(2)$ & $4.0(0.8)$ \\
\hline $574(4)$ & $-74(1)$ & $474(2)$ & $3.9(0.8)$ \\
\hline 608 (4) & $-47(1)$ & 389 (2) & $4.9(0.8)$ \\
\hline 647 (3) & $-138(1)$ & $567(1)$ & $5.4(0.6)$ \\
\hline $506(5)$ & $-131(2)$ & $642(3)$ & $6.7(1.0)$ \\
\hline $124(5)$ & $-145(2)$ & $603(3)$ & $5.0(1.0)$ \\
\hline $139(5)$ & $-87(2)$ & $610(3)$ & $6.0(1 \cdot 1)$ \\
\hline $160(5)$ & $-50(2)$ & $536(2)$ & $4.5(1.0)$ \\
\hline $177(5)$ & $-77(2)$ & 452 (3) & $5 \cdot 7(1.0)$ \\
\hline $171(5)$ & $-132(2)$ & 441 (2) & $4.4(0.9)$ \\
\hline $141(5)$ & $-170(2)$ & $516(3)$ & $5.5(1.0)$ \\
\hline $201(5)$ & $-26(2)$ & 385 (3) & $6.9(1 \cdot 1)$ \\
\hline $214(6)$ & $-43(2)$ & $317(3)$ & $9.6(1.3)$ \\
\hline $236(5)$ & $4(2)$ & $250(3)$ & $5.6(1.0)$ \\
\hline $226(5)$ & $62(2)$ & $272(3)$ & $6 \cdot 6(1 \cdot 1)$ \\
\hline $240(5)$ & $100(2)$ & $198(2)$ & $5 \cdot 3(1.0)$ \\
\hline $268(4)$ & $80(1)$ & $110(2)$ & $5.3(0.9)$ \\
\hline $275(4)$ & $22(1)$ & 90 (2) & $5.2(0.9)$ \\
\hline $257(5)$ & $-14(2)$ & $167(3)$ & $7.0(1.0)$ \\
\hline $276(3)$ & $121(1)$ & 40 (2) & $5.1(0.7)$ \\
\hline $356(4)$ & 108 (1) & $-45(2)$ & $4.2(0.8)$ \\
\hline 359 (4) & $148(1)$ & $-116(2)$ & $3.4(0.7)$ \\
\hline 294 (4) & 203 (1) & $-99(2)$ & $5.4(0.9)$ \\
\hline $302(4)$ & 237 (1) & $-172(2)$ & $4.2(0.8)$ \\
\hline 384 (4) & 221 (1) & $-262(2)$ & $4.1(0.8)$ \\
\hline $455(4)$ & 169 (1) & $-284(2)$ & $4.9(0.8)$ \\
\hline $434(4)$ & 134 (1) & -209 (2) & $4.7(0.8)$ \\
\hline 389 (3) & 261 (1) & $-328(2)$ & $5.9(0.6)$ \\
\hline $470(4)$ & 248 (1) & $-425(2)$ & $5.4(0.9)$ \\
\hline $121(5)$ & $-152(2)$ & $586(3)$ & $6.7(1.0)$ \\
\hline $137(5)$ & $-94(2)$ & $598(3)$ & $5 \cdot 4(1 \cdot 1)$ \\
\hline $156(5)$ & $-59(2)$ & $513(2)$ & $6 \cdot 3(1.0)$ \\
\hline $165(5)$ & $-92(2)$ & 436 (3) & $8.3(1.0)$ \\
\hline $156(5)$ & $-124(2)$ & $432(2)$ & $10.2(0.9)$ \\
\hline $129(5)$ & $-182(2)$ & 509 (3) & $7.6(1.0)$ \\
\hline $215(10)$ & $-59(3)$ & 344 (6) & $4.8(2 \cdot 1)$ \\
\hline $217(11)$ & $-8(4)$ & $353(6)$ & $9.4(2.4)$ \\
\hline $238(5)$ & $17(2)$ & $263(3)$ & $8 \cdot 7(1.0)$ \\
\hline $227(5)$ & $71(2)$ & 264 (3) & $10 \cdot 3(1 \cdot 1)$ \\
\hline $244(5)$ & $110(2)$ & $193(2)$ & $8 \cdot 1(1.0)$ \\
\hline $277(4)$ & 80 (1) & $105(2)$ & $6.0(0.9)$ \\
\hline $286(4)$ & 21 (1) & $95(2)$ & $5.9(0.9)$ \\
\hline $263(5)$ & $-13(2)$ & $177(3)$ & $7.7(1.0)$ \\
\hline
\end{tabular}


bone-like pattern. Along a, the molecular arrangement consists of alternate, antiparallel molecules of $A$ and $B$ extending infinitely.

In the structure there are 161 intermolecular contact distances less than $4 \AA$, but none of them is abnormally short. Molecule $A$ is surrounded by 14 neighbouring molecules with contact distances less than $4 \AA$. Molecule $B$, however, has only 10 neighbours and this difference in the number of nearest neighbours could be related to the disorder observed in molecule $B$.

The authors wish to thank Professor S. Chandrasekhar and Dr S. Ramaseshan for their kind interest.

\section{References}

Brown, C. J. (1966a). Acta Cryst. 21, 146-152.

Brown, C. J. (1966b). Acta Cryst. 21, 153-158.

Cromer, D. T. \& W Aber, J. T. (1965). Acta Cryst. 18, 104 109.

Germain, G., Main, P. \& Woolfson, M. M. (1971). Acta Cryst. A27, 368-376.

Hoekstra, A., Meertens, P. \& Vos, A. (1975). Acta Cryst. B31, 2813-2817.

Krigbaum, W. R. \& Taga, T. (1974). Mol. Cryst. Liq. Cryst. 28, 85-98.

ViJAYAn, K. \& VANI, G. V. (1975). Pramana Suppl. 1, 7578. 